\title{
Positronium Formation in Solid Long-Chain Alkanes
}

\author{
R. Zaleski, J. Wawryszczuk And T. Goworek \\ Institute of Physics, Maria Curie Skłodowska University \\ pl. Marii Curie-Skłodowskiej 1, 20-031 Lublin, Poland
}

This paper represents a fragment of systematic studies on positron annihilation in solid saturated hydrocarbons (alkanes, paraffins) from $\mathrm{C}_{17} \mathrm{H}_{36}$ to $\mathrm{C}_{30} \mathrm{H}_{62}$. The Tao-Eldrup model and its extensions allow to identify the places in the crystalline structure where positronium is trapped, and estimate respective trap sizes. In the rigid phase I positronium locates mainly in two-dimensional empty spaces between the lamellae of crystal structure. Additional free volumes exist in high temperature phases due to appearance of $n$-alkane conformers representing a non-planar molecule distortion. The rise of ortho-positronium intensity with the sample irradiation dose, which was seen earlier in polymers, is observed also in $n$-alkanes. One can find similarities between the ortho-positronium annihilation in crystalline alkanes and polymers, like exponential character of that rise, and the trap bleaching by illumination. It confirms the same origin of the intensity growth, i.e. the accumulation of trapped electrons. Two kinds of electron traps were found. The time constant of electron accumulation in the traps depends on the carbon chain length and varies with temperature in different way for even- and odd-numbered alkanes.

PACS numbers: 36.10.Dr, 78.70.Bj

\section{Introduction}

Solid long-chain $n$-alkanes are interesting subject of positron study. Crystallographic studies $[1,2]$ and infrared spectrometry [3, 4] give good knowledge of $n$-alkanes properties. At low temperature in the rigid phase straight hydrocarbon molecules, parallel to each other, are ordered in lamellar structure. Only the longitudinal (screw-like) molecule movements are possible. When closing to the melting temperature variety of phases is observed depending on alkane chain length (Fig. 1). The phases differ by molecule orientations or inter-lamellar shift. A few 


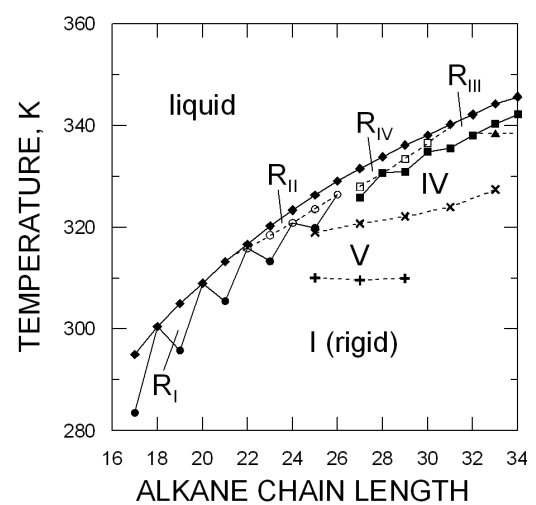

Fig. 1. Phase diagram for $n$-alkanes $[1,2]$.

degrees below the melting point the rotator phases appear. Beside molecule motion the molecular shape is distorted comparing to "all-trans" conformer. Concentrations of each non-planar conformer ("end-gauche", "double-gauche" and "kink") vary depending on temperature and phase. These distortions result in the appearance of free volumes where positronium can be created and trapped. Positron annihilation lifetime spectroscopy (PALS) measurements shows ortho-positronium (o-Ps) intensity walk similar to that observed before in polymers e.g. polyethylene [5]. It is not surprising because the only difference between these compounds lies in the length of carbon chain: both represent repeating series of $\mathrm{CH}_{2}$ groups with $\mathrm{CH}_{3}$ on the ends. Simple, well defined structure of $n$-alkanes gives an opportunity to understand better the origin of $o$-Ps intensity rise.

\section{Experimental}

Investigated samples $\left(\mathrm{C}_{n} \mathrm{H}_{2 n+2}\right.$, where $\left.n=17 \div 30\right)$ from Aldrich were $99 \%$ or better purity as confirmed by gas chromatography. The PAL spectra were measured using a conventional fast-slow delayed coincidence spectrometer with $\mathrm{BaF}_{2} \gamma$ detectors. Its resolution was about $210 \mathrm{ps}, \mathrm{FWHM}$. The statistics collected per one spectrum during about two hours was of order of $10^{6}$ counts. The sample was surrounding $0.8 \mathrm{MBq}{ }^{22} \mathrm{Na}$ positron source in kapton envelope (correction: $10 \%, 374 \mathrm{ps})$. Such a sandwich was placed in a small brass container fixed at a copper rod inside the vacuum chamber. The lower end of the copper rod was submerged in liquid nitrogen and temperature was stabilized by heating the upper end. Temperature change and stabilization were performed by a programmed controller. Before the measurement the sample was "annealed" at the temperature close to the melting point. Then usually the sample was cooled at rate of about $6 \mathrm{~K} / \mathrm{min}$ to initial low temperature. Afterwards the temperature was changed stepwise and stabilized during spectra collection. Three discrete components were fitted to the spectra using the LT programme [6]. According to the statistical 
weights of the para- and ortho-states, intensity ratio was fixed 1:3. Detection of fast (more than one hour is needed to collect good statistics per spectrum) changes of ortho-positronium intensity was possible by using the multiscaler mode. Instead of analyzing the whole spectrum one can pay attention on the sum of coincidences corresponding to the long-lived part of the time spectrum. It can be realized by count summation in the window chosen for lifetime spectra measured in a short time. More direct way is connecting the output of time-to-amplitude converter to a single channel analyzer (SCA). The SCA selects the chosen range of amplitudes and sends the pulses to the multichannel analyzer working in a multiscaler mode. Sufficient statistics per channel can be collected in one minute or less. If the lifetime $\tau_{3}$ is constant the number of counts is proportional to $I_{3}$.

\section{Results and discussion}

\subsection{Phases and molecule distortions}

At the low temperatures (phase I) the ortho-positronium lifetime $\tau_{3}$ shows almost linear slowly rising temperature dependence for all investigated $n$-alkanes (Fig. 2). It is seen in Fig. 3 that odd-numbered hydrocarbons have the lifetimes about 200-300 ps longer than even-numbered ones. Crystallographic studies done by Denicolo et al. [1] show that inter-lamellar spacing in odd-numbered alkanes is $0.195 \mathrm{~nm}$, while in even ones - $0.125 \mathrm{~nm}$ at the room temperature. It allows to identify inter-lamellar spaces as the free volumes where positronium is trapped. For such one-dimensional spaces at the room temperature the extended Tao-Eldrup (ETE) model [7] predicts rather $\tau_{\text {3even }}^{\mathrm{ETE}} \approx 1.0 \mathrm{~ns}$ and $\tau_{3 \mathrm{odd}}^{\mathrm{ETE}} \approx 1.4 \mathrm{~ns}$ than $\tau_{3 \text { odd }}^{\text {exp }} \approx$ $1.3 \mathrm{~ns}$ and $\tau_{3 \mathrm{odd}}^{\exp } \approx 1.5 \mathrm{~ns}$ obtained from the experiment. In the rigid phase the only way of growing free volumes are longitudinal molecule motions. Such moves result in local growth of inter-lammelar space thickness resulting in lifetimes longer than expected.

Smooth lifetime changes with temperature rise are perturbed in a range of a few degrees below the melting point. Appearance of rotator phases reflects in rapid lifetime rise, except $\mathrm{C}_{18} \mathrm{H}_{38}$ and $\mathrm{C}_{20} \mathrm{H}_{42}$ where rotator phase is not observed during heating. Lifetime rise is well correlated to concentration of non-planar conformers $[3,4]$. This suggests that a free volume, where positronium annihilates, appears in the neighbourhood of distorted molecule. At low temperature the alkane crystal consists of rod-like molecules, where $\mathrm{C}-\mathrm{C}$ bonds are arranged in a zig-zag form ("all-trans" conformer). Conformers "end-gauche" and "doublegauche" are the molecules bent at second or third carbon due to turning $\mathrm{C}-\mathrm{C}$ bond to opposite side (gauche bond). "Kink" distortion is combination of two bends forming two parallel but shifted fragments of a molecule connected by kink. The kink is able to move along a molecule. In all cases the shape of free volume is oblong and can be approximated by a cylinder in model calculations. Observed rotator phase lifetimes becomes longer with carbon chain elongation closing to the 


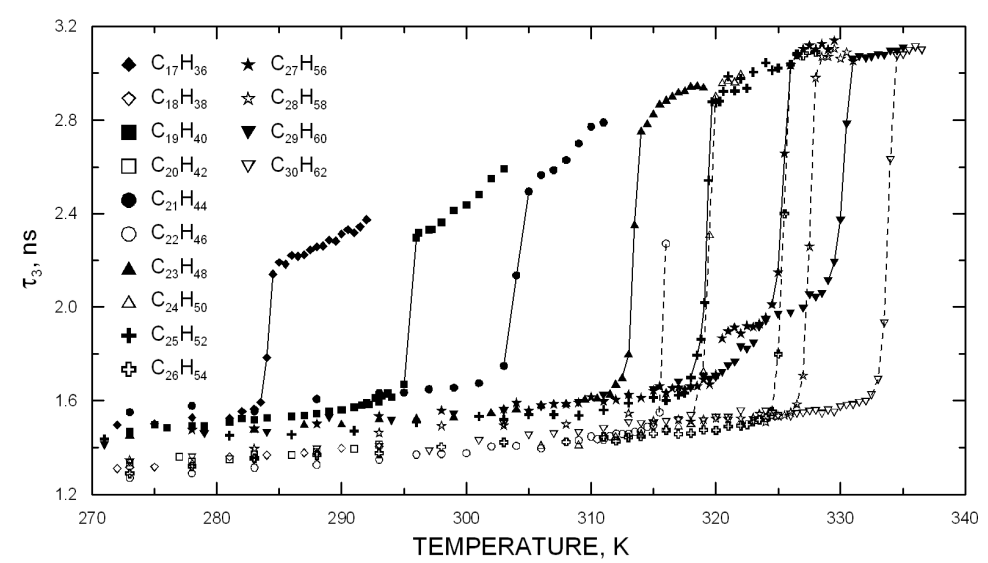

Fig. 2. Ortho-positronium lifetime as a function of temperature for $n$-alkanes from $\mathrm{C}_{17} \mathrm{H}_{36}$ to $\mathrm{C}_{30} \mathrm{H}_{62}$.

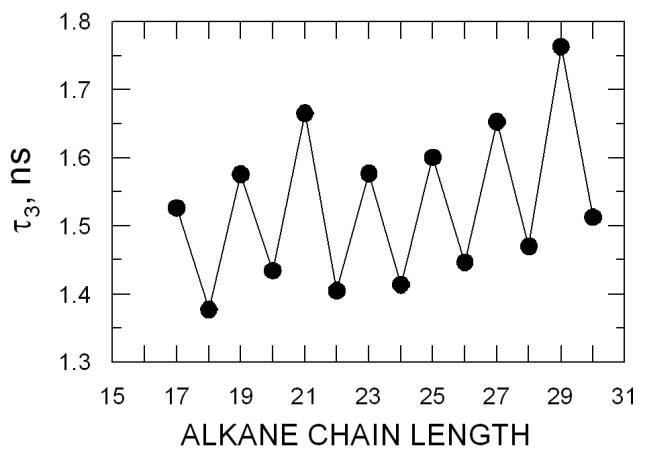

Fig. 3. Ortho-positronium lifetime 15 degrees below the melting point for respective $n$-alkanes.

saturation value $\approx 3.1 \mathrm{~ns}$ (Fig. 2) for $n>26$. Assuming that the positronium trap near "kink" molecule is about half molecule length long one can estimate the cross-section of free volume using ETE model and experimental lifetime saturation value. Obtained result $0.32 \mathrm{~nm}^{2}$ is over 1.5 times more than the section of "all-trans" molecule which is $0.19 \mathrm{~nm}^{2}$. Using this result one can calculate relative length of the cylindrical free volume corresponding to the lifetimes of studied alkanes (Fig. 4). The influence of inter-lamellar space leading to overestimating the length values was neglected. The dependence between relative trap length and chain length changes its character when phases $R_{\mathrm{I}}$ and $R_{\mathrm{II}}$ are replaced by $R_{\mathrm{III}}$ and $R_{\mathrm{IV}}$ (see Fig. 1). Similar calculations were done for lifetimes making additional "step" at the temperature region of phase $\mathrm{IV}$ in $\mathrm{C}_{27} \mathrm{H}_{56}$ and $\mathrm{C}_{29} \mathrm{H}_{60}$. Such lifetimes correspond to free volumes close to these produced by "end-gauche" and "double-gauche" distortion with no "kink" defects. That is a confirmation of the results obtained by Snyder et al. [8]. No other phases were distinguished during 


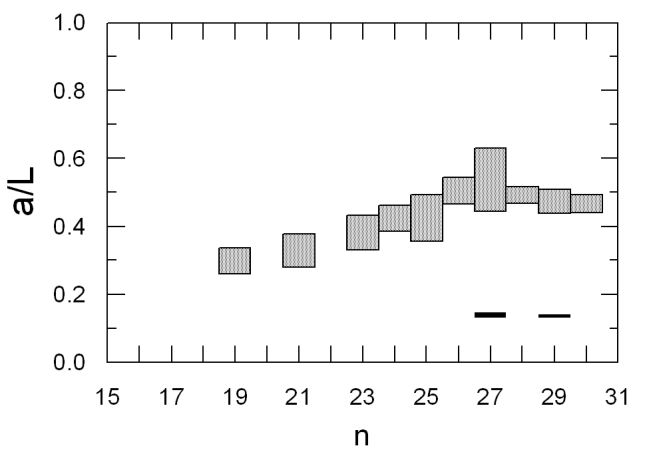

Fig. 4. Relative length of the cylindrical free volume $(R=0.32 \mathrm{~nm}, L=n \times 0.1273 \mathrm{~nm})$ expected in rotator phase basing on extended Tao-Eldrup model predictions for investigated $n$-alkanes. Black bars are relative lengths calculated for phase IV lifetimes.

sample heating, however supercooling makes phase transition shifts sufficiently large to observe additional phases; in $\mathrm{C}_{27} \mathrm{H}_{56}$ phase $\mathrm{V}$ can be noticed, in $\mathrm{C}_{25} \mathrm{H}_{52}$ phase IV appears.

\subsection{Intensity walk}

Ortho-positronium formation probability rises with time during PALS measurements in $n$-alkanes. Similar effect was observed in polyethylene. It was explained by Wang et al. [5] as a result of accumulation of trapped electrons due to positron sample irradiation. At low doses typical feature is the exponential-like growth of the intensity in the darkness and its rapid decrease after a visible light turning on. Similar measurements for $n$-alkanes were performed [9]. Dose rate provided by the standard PALS source was less than $0.1 \mathrm{~Gy} / \mathrm{h}$ and free radical related intensity decrease, present in polyethylene at dose rate of order $\mathrm{kGy} / \mathrm{h}[10]$, was not observed in $n$-alkanes. In both cases intensity drops when incandescent light is on confirming the same origin of intensity walk. A series of measurements of intensity rise time constant was done for investigated $n$-alkanes at temperatures in the range $100 \mathrm{~K}$ to $300 \mathrm{~K}$. Long time constants $(\Theta \approx 30-70 \mathrm{~h})$ rising with temperature were seen in even-numbered alkanes, while shorter $(\Theta \approx 10 \mathrm{~h})$ decreasing to one hour in odd-ones. At increasing temperature step-by-step in even-numbered alkanes the intensity $I_{3}$ rises reaching maximum at about $200 \mathrm{~K}$, and then decreases stabilizing at certain level. Fast changes occurring after temperature changes in odd alkanes can be observed using multiscaler mode as shown in Fig. 5. After a fast cooling from rotator phase to highest intensity region the number of counts rises with time constant $\Theta=2.4 \mathrm{~h}$. It is comparable to the spectrum collection time, so step-by-step measurement may give underestimated values of intensity in this region. Next temperature change from $200 \mathrm{~K}$ to $250 \mathrm{~K}$ causes ortho-positronium intensity to fall to the value obtained near room temperature plateau. The time constant of the intensity decrease from saturation value 


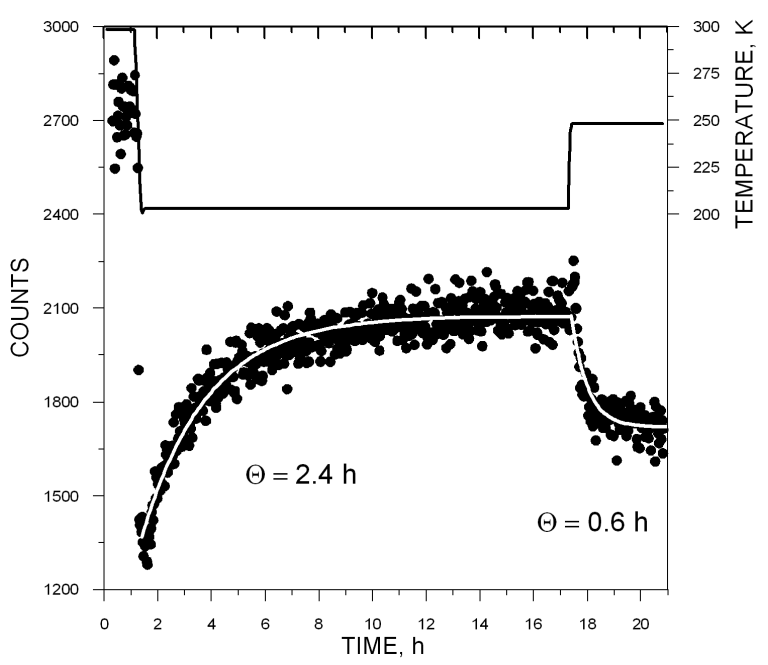

Fig. 5. Multiscaler results for ortho-positronium intensity walk (black dots) in $\mathrm{C}_{19} \mathrm{H}_{40}$ after cooling form rotator phase to $200 \mathrm{~K}$ and after heating from $210 \mathrm{~K}$ to $250 \mathrm{~K}$, white lines are exponential functions fitted, black line shows temperature changes during measurement.

in $200 \mathrm{~K}$ after fast heating from $200 \mathrm{~K}$ to $250 \mathrm{~K}$ is much shorter than one of the step-by-step measurements. Basing on this result the intensity values in this temperature range can be assumed as saturation values. The $I_{3}$ changes in this region can be interpreted as the result of disappearance of the electron traps. It may occur due to thermal activation of molecular motions, as suggested by Hirade [11] in the case of polymers. Enthalpy needed for the traps removal can be estimated by drawing the Arrhenius plot. Such calculations for $\mathrm{C}_{19} \mathrm{H}_{40}[12]$ and $\mathrm{C}_{25} \mathrm{H}_{52}$ [13] give similar enthalpy value $H=0.6 \mathrm{eV}$. The intensity $I_{3}$ above $250 \mathrm{~K}$ is still unstable; the time constant of this walk is of the order of one hour. This effect was observed up to a few degrees below the rotator phase transition temperature indicated by the lifetime change as shown in Fig. 2. It suggests the existence of another kind of electron traps. Second kind of the traps seems to remain active, i.e. trapping electrons, also at low temperatures. It may be tested by approximating $I_{3}$ time dependence by the sum of two exponentials (1) in this temperature region because in the presence of two kinds of electron traps one should observe contribution from each of them to the intensity rise. The obtained curve fits better initial fast $I_{3}$ rise, where dominates component with $\Theta=1-2 \mathrm{~h}$, that suits the time constant value observed above $250 \mathrm{~K}$ :

$$
I_{3}=I_{3}^{0}+I_{3 \operatorname{trap}}^{(1)}\left[1-\exp \left(-t / \Theta_{1}\right)\right]+I_{3 \operatorname{trap}}^{(2)}\left[1-\exp \left(-t / \Theta_{2}\right)\right] .
$$




\section{Conclusions}

Observing phase transitions in long-chain $n$-alkanes using the PALS gives opportunity to confront extended Tao-Eldrup predictions with well known properties of these substances. Molecule shape alteration resulting in o-Ps lifetime change allows estimating free volume sizes. The results are in agreement with infrared spectrometry data and can be explained by appearance of various nonplanar conformers ("end-gauche", "double-gauche" and "kink") concentrations. During sample cooling run the supercooling effect occurs, additional phases (not distinguishable during heating) were noticed.

Ortho-positronium intensity walk (rise) was observed. Light bleaching effect [9] confirms its origin as the same as in polymers where it was explained by positron irradiation induced electron trapping. Two kinds of the traps were found. Activation enthalpy of one of them was estimated to be $0.6 \mathrm{eV}$. Well known for $n$-alkanes odd-even differences were seen in the PAL spectra: the difference in crystalline structure (inter-lamellar space) reflects in ortho-positronium lifetime values and its intensity rises in a different way depending on carbon number evenness.

\section{References}

[1] I. Denicolo, J. Doucet, A.F. Craievich, J. Chem. Phys. 78, 1465 (1983).

[2] K. Nozaki, N. Higashitani, T. Yamamoto, T. Hara, J. Chem. Phys. 103, 5762 (1995).

[3] M. Maroncelli, Song Ping Qi, H.L. Strauss, R.G. Snyder, J. Am. Chem. Soc. 104, 6237 (1982).

[4] Yesook Kim, H.L. Strauss, R.G. Snyder, J. Phys. Chem. 93, 7520 (1989).

[5] C.L. Wang, T. Hirade, F.H.J. Maurer, M. Eldrup, N.J. Pedersen, J. Chem. Phys. 108, 4654 (1998).

[6] J. Kansy, Nucl. Instrum. Methods Phys. Res. A 374, 235 (1996).

[7] B. Jasinska, A.E. Kozioł, T. Goworek, J. Radioanal. Nucl. Chem. 210, 617 (1996).

[8] R.G. Snyder, M. Maroncelli, Song Ping Qi, H.L. Strauss, Science 214, 188 (1981).

[9] N. Djourelov, T. Goworek, K. Kondo, T. Suzuki, R. Zaleski, Phys. Lett. A 323, 165 (2004).

[10] T. Hirade, F.H.J. Maurer, M. Eldrup, Rad. Phys. Chem. 58, 465 (2000).

[11] T. Hirade, Mater. Sci. Forum 445-446, 234 (2004).

[12] T. Goworek, R. Zaleski, J. Wawryszczuk, Chem. Phys. 295, 243 (2003).

[13] B. Zgardzinska, R. Zaleski, J. Wawryszczuk, T. Goworek, Phys. Lett. A 333, 341 (2004). 